\title{
The dynamic resistance of YBCO coated conductor wire: Effect of DC current magnitude and applied field orientation
}

\author{
Zhenan Jiang ${ }^{1}$, Wei Zhou ${ }^{1,2}$, Quan $\mathrm{Li}^{3}$, Min Yao ${ }^{3}$, Jin Fang ${ }^{2}$, Naoyuki Amemiya ${ }^{4}$, Chris \\ W. Bumby ${ }^{1}$ \\ ${ }^{1}$ Robinson Research Institute, Victoria University of Wellington, PO BOX 33436, Petone, \\ Lower Hutt 5046, New Zealand \\ ${ }^{2}$ School of Electrical Engineering, Beijing Jiaotong University, Haidian, Beijing 100044, \\ The People's Republic of China \\ ${ }^{3}$ School of Engineering, University of Edinburgh, Faraday Building, The King's Buildings, \\ Edinburgh EH9 3JL, UK \\ ${ }^{4}$ Department of Electrical Engineering, Graduate School of Engineering, Kyoto University, \\ Kyoto-Daigaku-Katsura, Nishikyo, Kyoto 615-8510, Japan \\ E-mail: zhenan.jiang@vuw.ac.nz
}

\begin{abstract}
Dynamic resistance, which occurs when a HTS coated conductor carries a DC current under an AC magnetic field, can have critical implications for the design of HTS machines. Here, we report measurements of dynamic resistance in a commercially available SuperPower $4 \mathrm{~mm}$-wide YBCO coated conductor, carrying a DC current under an applied AC magnetic field of arbitrary orientation. The reduced DC current, $I_{\mathrm{t}} / I_{\mathrm{c} 0}$, ranged from 0.01 to 0.9 , where $I_{\mathrm{t}}$ is the DC current level and $I_{\mathrm{c} 0}$ is the self-field critical current of the conductor. The field angle (the angle between the magnetic field and the normal vector of the conductor wide-face) was varied between $0^{\circ}$ and $90^{\circ}$ at intervals of $10^{\circ}$. We show that the effective width of the conductor under study is $\sim 12 \%$ less than the physical wire width, and we attribute this difference to edge damage of the wire during or after manufacture. We then examine the measured dynamic resistance of this wire under perpendicular applied fields at very low DC current levels. In this regime we find that the threshold field, $B_{\text {th }}$, of the conductor is well described by the non-linear equation of Mikitik and Brandt. However, this model consistently underestimates the threshold field at higher current levels. As such, the dynamic resistance in a coated conductor under perpendicular magnetic fields is best described using two different equations for each of the low and high DC current regimes. At low DC currents where $I_{\mathrm{t}} / I_{\mathrm{c} 0} \leq 0.1$, the non-linear relationship of Mikitik and Brandt provides the closest agreement with experimental data. However, in the higher current regime where $I_{t} / I_{\mathrm{c} 0} \geq 0.2$, closer agreement is obtained using a simple linear expression which assumes a current-independent penetration field. We further show that for the conductor studied here, the measured dynamic resistance at different field angles is dominated by the perpendicular magnetic field component, with negligible contribution from the parallel component. Our findings now enable the dynamic resistance of a single conductor to be analytically determined for a very wide range of DC currents and at all applied field angles.
\end{abstract}

Keywords: Dynamic resistance, AC loss, YBCO, REBCO

\section{Introduction}


The current carrying performance of REBCO coated conductors has increased significantly in recent years, and has reinvigorated interest in HTS power applications utilising REBCO wires [1]. Many HTS machinery applications require an HTS wire to carry DC current whilst experiencing an applied alternating magnetic field, and in these applications dynamic resistance plays critical role [2-5]. HTS field windings in rotating machines experience an applied ripple field whilst carrying a DC current. The resulting dynamic resistance leads to heat dissipation which may impose a substantial heat load on the cryogenic system [6-8]. Dynamic resistance is also critical to the design and operation of HTS flux pumps, as it sets a limit to the maximum achievable current which such devices can deliver [9-13]. Despite these varied applications, there have been limited reports of the measurement of dynamic resistance in practical HTS coated conductors.

Experimental reports to date have focused on dynamic resistance measurements in REBCO coated conductors under perpendicular AC magnetic fields [14-17]. Several analytical equations have been developed to predict dynamic resistance in this geometry $[16,18]$. If we define a thin superconductor as seen in figure 1, the dynamic resistance per unit length per cycle in a perpendicular AC magnetic field can be written as $[16,18]$,

$$
\frac{R_{\mathrm{dyn}, \perp}}{f L}=\frac{4 a}{I_{\mathrm{c} 0}}\left(B_{\mathrm{a}, \perp}-B_{\mathrm{th}, \perp}\right),
$$

where, $a$ is half-width of the conductor, $I_{\mathrm{c} 0}$ is the self-field critical current of the conductor, $B_{\mathrm{a}, \perp}$ is the magnetic field amplitude. Recently $[16,17]$ we have suggested that the threshold field, $B_{\mathrm{th}, \perp}$, can be expressed as

$$
B_{\mathrm{th}, \perp}=B_{\mathrm{p}, \perp}(1-i)=\frac{4.9284 \mu_{0} J_{\mathrm{co} 0} t}{\pi}(1-i),
$$

where, $i$ is $I_{\mathrm{t}} / I_{\mathrm{c} 0}, J_{\mathrm{c} 0}$ is critical current density and $t$ is half-thickness of the conductor. $B_{\mathrm{p}, \perp}$ is the effective penetration field which can be obtained from the maxima of the Brandt equation for AC magnetization loss [19]. It has been shown equation (2) provides good agreement with all previously-published experimental data for $i>0.1$ [16]. However, an alternative expression for the threshold field is provided by Mikitik and Brandt, $B_{\text {th,MB }}[18]$,

$$
B_{\mathrm{th}, M \mathrm{~B}}=\frac{\mu_{0} J_{\mathrm{c} 0} t}{2 \pi}\left[\frac{1}{i} \ln \left(\frac{1+i}{1-i}\right)+\ln \left(\frac{1-i^{2}}{4 i^{2}}\right)\right] .
$$

Equations 2 and 3 diverge markedly in the low current region, and this is therefore the most sensitive region to discern between the applicability of each expression. However, until now, there have been no reported measurements of dynamic resistance for which $i<0.1$. In addition, in actual HTS coated conductor applications, the conductors are exposed to AC magnetic fields with non-perpendicular orientations. To date, there is only one previous report of the effect of varying the applied AC field angle on the measured dynamic resistance [17], and this was limited to only three different orientations. In this work, we have addressed both of these gaps in the literature. We have measured the dynamic resistance of a $4 \mathrm{~mm}$-wide YBCO coated conductor manufactured by SuperPower (SCS4050), using our newly developed measurement system [20]. We have studied a wide range of $i$ values, including finely resolved data for $i<0.1$. The field angle was varied from $0^{\circ}$ to $90^{\circ}$ at a resolution of $10^{\circ}$. The measured dynamic resistance was compared with the analytical expressions above, as well as to $2 \mathrm{D}$ finite element calculations using the $T$ method described in chapter 3 of [21]. 


\section{Experimental methods}

The measurement of dynamic resistance was undertaken using a similar method to that previously reported $[17,22]$. A custom made AC magnet comprising two halves of racetrack coils was used to generate more than $100 \mathrm{mT}$ peak amplitude at three different frequencies [20]. The $15 \mathrm{~cm}$-long sample was mounted on the machined surface of a G-10 sample holder and was inserted into the magnet bore. The field angle of the sample was adjusted by rotating the sample holder whilst monitoring a one-turn pick-up coil on the flat surface of the sample holder. Current leads were arranged in order to avoid magnetic coupling as shown in figure 2(a). A DC power supply (Hewlett Packard 6682A) was used to feed DC current to the sample. The coated conductor sample was fabricated by SuperPower (SCS4050), and has a self-field $I_{\mathrm{c}}$ of $87.7 \mathrm{~A}$ at $77 \mathrm{~K}$. Key specifications of the sample are shown in Table 1. Figure 2(b) shows the arrangement of two sets of voltage taps attached to the sample: a spiral loop was arranged around the sample for one set; whilst the second set comprised two voltage taps attached at the centre of the sample and extended from their midpoint $[18,23]$. The distance between the voltage-taps was $5 \mathrm{~cm}$. The voltage output from the voltage taps were measured using a DC nanovolt-meter (Hewlett Packard 34420A). The integration time (NPLC) of the nanovolt-meter was set at 10 power line cycles. Data obtained from the two sets of voltage taps was found to be almost identical, so data from the spiral loop taps has been reported throughout this work.

\section{Numerical methods}

The local current and magnetic flux are key factors to determine losses in HTS coated conductors. $T$ formulation is a finite-element electromagnetic modelling approach which was developed to help clarify how current and magnetic flux varies and affects losses within 2D superconducting films [24-26]. This method has been broadly validated and applied in quantifying losses [27-30]. However, the ac loss calculated by this approach is a total loss which includes both magnetisation loss and dynamic loss (i.e. that due to dynamic resistance). In this paper we have amended the $T$-model to enable these two different sources of loss to be separated, so that a calculated dynamic resistance can be compared to our experimental results. The thin-strip approximation was applied, since HTS coated conductors comprise very thin films of superconducting material with a very high aspect ratio. The governing equations are formulated using the current vector potential $T$ instead of the current density $J$, which is defined by:

$$
J=\nabla \times T .
$$


The total AC loss per unit volume can be calculated from:

$$
P=J E=\frac{J^{2}}{\sigma_{s c}}
$$

where $\sigma_{\mathrm{sc}}$ is conductivity of the superconductor layer. When an HTS coated conductor carries a DC transport current under an AC magnetic field, the transport current $I_{\mathrm{t}}$ will occupy the central region of the superconducting film, within a distance $2 a i$ in the centre of the coated conductor, leaving the rest with width 2(1-i) $a$ free on both sides [16]. Consequently, the dynamic loss $P_{\text {dyn }}$ (which is the loss due solely to dynamic resistance) may be approximately calculated by:

$$
P_{\mathrm{dyn}}=\int_{(1-i) a}^{(1+i) a} 2 J E t \mathrm{~d} y=\int_{(1-i) a}^{(1+i) a} \frac{J^{2}}{\sigma_{s c}} 2 t \mathrm{~d} y
$$

where $i$ is the ratio between transport current $I_{\mathrm{t}}$ and self-field critical current $I_{\mathrm{c}}$ and $t_{\mathrm{s}}$ is the thickness of the superconductor layer.
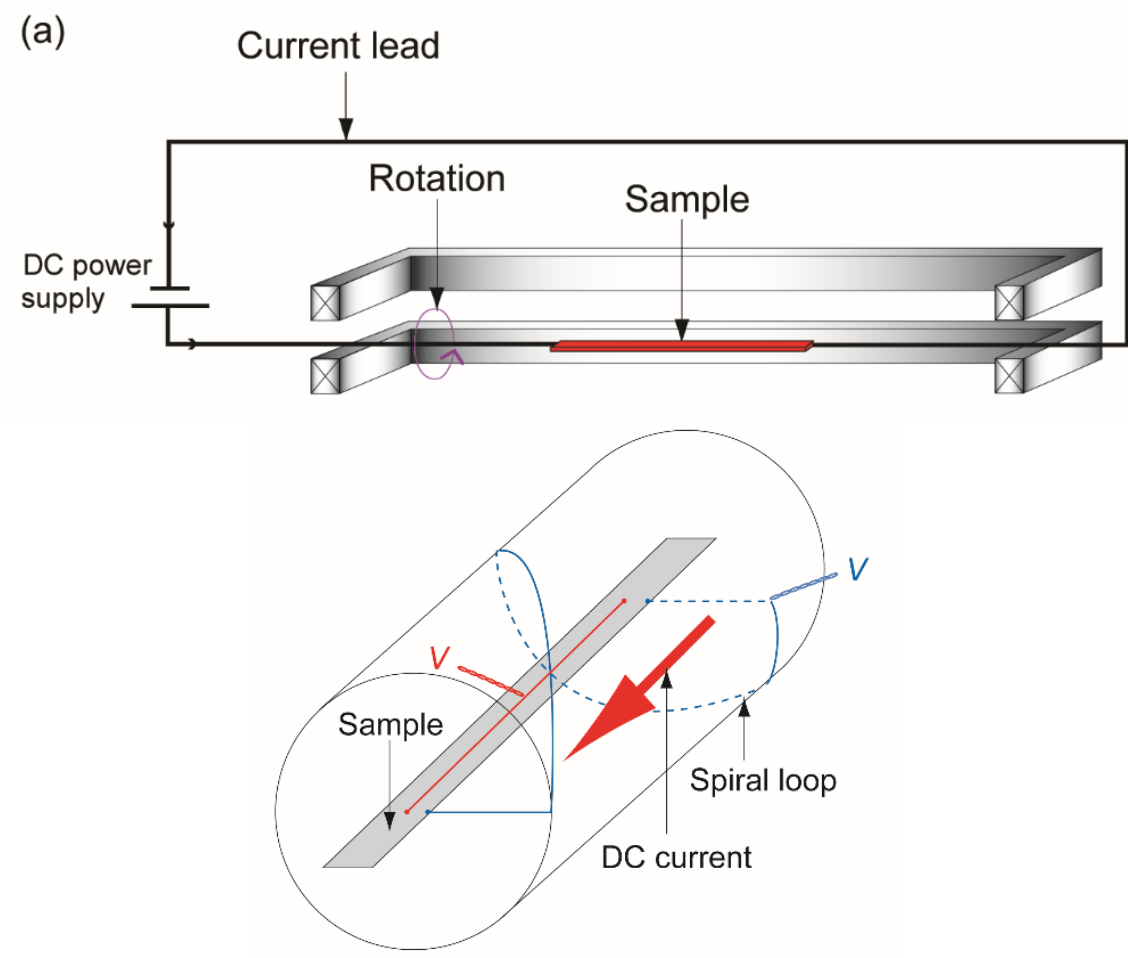

Figure 2. (a) Schematic of the measurement and (b) schematic of voltage tap arrangement 
Table 1 YBCO conductor specifications

\begin{tabular}{|c|c|}
\hline Manufacturer & SuperPower \\
\hline Self-field critical current (A) & $87.7 \mathrm{~A}$ \\
\hline Sample width (mm) & 4.0 \\
\hline Thickness of $\mathrm{Cu}$ stabilizer layer each side $(\mu \mathrm{m})$ & 20 \\
\hline Thickness of superconductor layer $(\mu \mathrm{m})$ & 1.0 \\
\hline Thickness of substrate $(\mu \mathrm{m})$ & 50 \\
\hline
\end{tabular}

\section{Experimental results and discussion}

\subsection{Measurements of $R_{d y n}$ under alternating perpendicular magnetic fields}

Figure 3 shows the measured $R_{\text {dyn }}$ values for the sample at two different frequencies and for five different values of reduced current, $i=I_{\mathrm{t}} / I_{\mathrm{c} 0}$. The $R_{\mathrm{dyn}}$ values are normalised by frequency and the sample length, and are plotted as a function of the amplitude of the applied alternating perpendicular magnetic field, $B_{\mathrm{a}, \perp}$. In the figure, analytical values calculated from equations (1) and (2), are also plotted, along with the results of the 2D FEM computation via the $T$ method.

For $i<0.7$, data obtained at both frequencies falls upon a single line which intercepts the $x$-axis at the threshold magnetic field, $B_{\mathrm{th}, \perp}$. The near-perfect agreement between data obtained at different frequencies reflects the hysteretic nature of dynamic resistance. For $i=0.9$, a linear correlation is observed at applied fields up to $\sim 60 \mathrm{mT}$, but at higher field amplitudes an additional non-linear contribution becomes apparent. We have previously shown that this non-linear component arises due to flux flow resistance, which occurs once the applied current exceeds the field-dependent critical current of the wire for part of the cycle (i.e. once 4 at $\left.\cdot J_{\mathrm{c}}\left(B_{\mathrm{a}, \perp}\right)<I_{\mathrm{t}}\right)$.

Experimental values for the threshold magnetic field, $B_{\mathrm{th}, \perp}$ were determined by fitting the linear section of the data shown in each plot in figure 3. $B_{\mathrm{th}, \perp}$ values are found to decrease with increasing DC current, as observed in previous works $[16,17]$. Notably, analytically values calculated from equations (1) and (2) were observed to slightly overestimate the experimental data, showing poorer agreement than our previous report on a different SuperPower wire [16]. We observe disagreement in both $B_{\mathrm{th}, \perp}$ and the gradient, $\mathrm{d} R_{\mathrm{dyn}} / \mathrm{d} B_{\mathrm{a}, \perp}$. The numerically computed 2D-FEM results broadly agree with the analytically calculated values, but also deviate from the experimental data. 


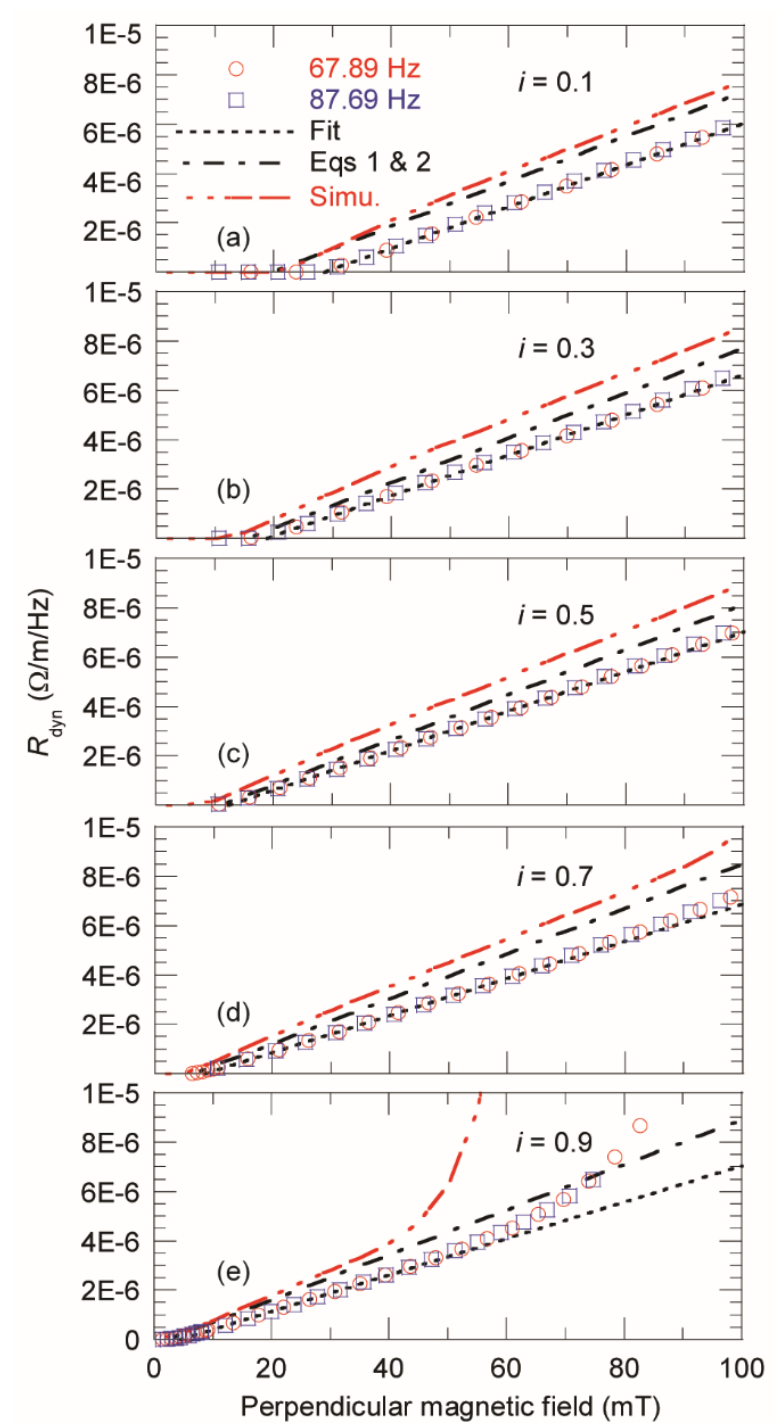

Figure 3. Measured $R_{\text {dyn }}$ values for the sample for $i=0.1,0.2,0.3,0.5,0.7$, and 0.9 at $67.89 \mathrm{~Hz}$ and $87.69 \mathrm{~Hz}$ compared with values derived from the combination of equations (1) and (2), and from FEM simulation using the $T$-formulation. 
To probe this discrepancy, figure 4 plots the gradients, $\mathrm{d} R_{\mathrm{dyn}} / \mathrm{d} B_{\mathrm{a}, \perp}$ obtained from each of the linear fits in figure 3 . These are plotted as a function of $I$, and compared with the theoretically expected value $4 a / I_{\mathrm{c} 0}$ from equation (1). The experimentally-obtained values are found to be consistent, but significantly lower than the theoretical value. We believe this difference to be due to edge effects in the coated conductor wire, resulting in an actual conductor width which is significantly smaller than the physical dimension of the wire (see figure 6). In coated conductors, the $J_{\mathrm{c}}$ values at each edge of the conductor are often found to be smaller than in the central region, due to damage caused by cutting processes [31-34]. If the true conductor width, $2 a$, is actually thinner than given in Table 1 , then this would explain why, in this case, both analytical and FEM calculations have under-estimated $B_{\mathrm{th}, \perp}$ and hence overestimated $R_{\text {dyn }}$.

Effective conductor widths are normally $90 \%$ of the conductor width due to slitting processes [38, 39]. If we take the average value of the experimentally determined gradient values, $\mathrm{d} R_{\mathrm{dyn}} / \mathrm{d} B_{\mathrm{a}}$, the difference between the average value and the analytical vale is $12.2 \%$, which implies an effect conductor width of 3.51 $\mathrm{mm}$. This assumes a uniform lateral $J_{\mathrm{c}}$ distribution such as shown in figure 6. (Typically lateral $J_{\mathrm{c}}$ distribution are trapezoidal, however here we have assumed a simpler pattern for convenience [32-34, 36, 37].)

Further evidence for a decreased effective conductor width is found in transport AC loss measurements of the same coated conductor wire. This data is shown in figure 5, where it is plotted as a function of the amplitude of the transport current. The measured sample was cut from the same source material as the sample used in the work. $I_{\mathrm{c} 0}$ of this short-length sample was $86.7 \mathrm{~A}$. In the figure, Norris strip and ellipse models (N-s and N-e in the figure) are plotted together [35]. The significant increase of the measured results above that predicted by N-s again implies a decreased effective conductor width, as has been discussed in previous works $[36,37]$.

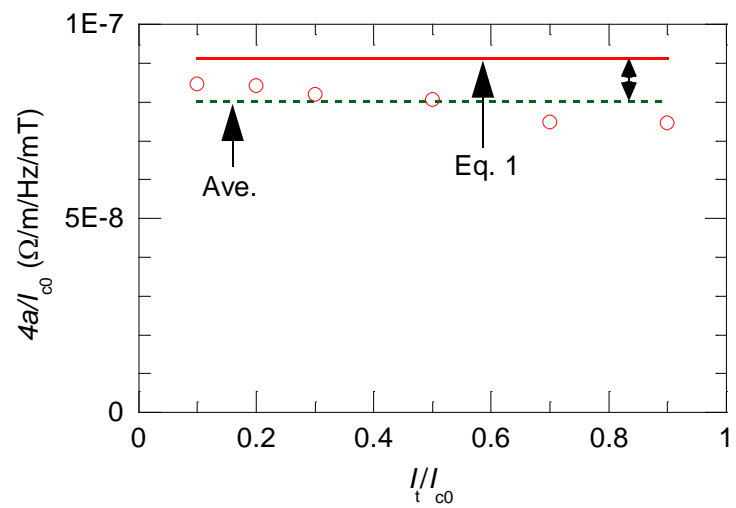

Figure 4. Comparison of the gradient of the linear fits, $\mathrm{d} R_{\mathrm{dyn}} / \mathrm{d} B_{\mathrm{a}}$ vs $4 a / I_{\mathrm{c} 0}$. 


\section{Figure 5. Transport AC loss of a YBCO conductor at $27.74 \mathrm{~Hz}$. The sample is cut from the same source} material as the sample used in the work. $I_{\mathrm{c} 0}$ of the sample is $86.7 \mathrm{~A}$.

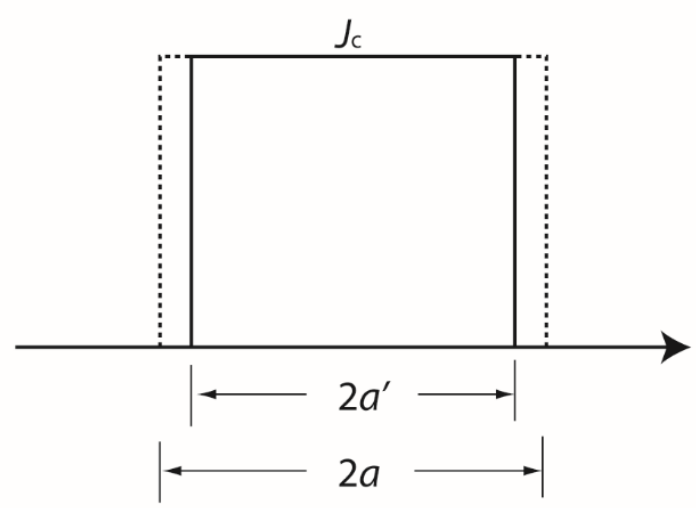

Figure 6. A schematic of assumed lateral $J_{\mathrm{c}}$ distribution and the effective conductor width. In this figure, $2 a$, $2 a$ stand for the physical conductor width and effective conductor width, respectively.

In light of this we have recalculated the analytical and FEM values using an effective conductor width of, $2 a$ $=3.51 \mathrm{~mm}$ and replotted this alongside the previously shown experimental values for $R_{\mathrm{dyn}}$. It is clear that the agreement between the measured data and equations (1) and (2) is now excellent for all linear regions shown. Interestingly the agreement between the measured data and 2D-FEM results is not quite as good as that between the measured data and the analytical results. Nonetheless, the agreement with the linear regime is still substantially improved over figure 3 . It is interesting to note that at $i=0.9$, the $2 \mathrm{D}$-FEM model substantially over-estimates the 'lift-off' due to non-linear flux-flow resistance at high magnetic fields, and thus deviates substantially from the experimental data [16]. The magnitude of the flux-flow resistance is highly sensitive to the $J_{\mathrm{c}}\left(B_{\mathrm{a}, \perp}\right)$ behaviour of the wire, and one possible cause of the strong disagreement in this region is that the 2D-FEM calculation used here assumed a constant $n$ value for all magnitudes of applied $B$. Another potential issue is that a key assumption in the FEM calculations performed here is that transport current flows only in the central region (see equation 6). This assumption may not necessarily be valid for those parts of the cycle where $I_{\mathrm{t}} \gg>I_{\mathrm{c}}\left(B_{a}\right)$. 
1

2

3

4

5

7

8

9

10

11

12

13

14

15

16

17

18

19

20

21

22

23

24

25

26

27

28

29

30

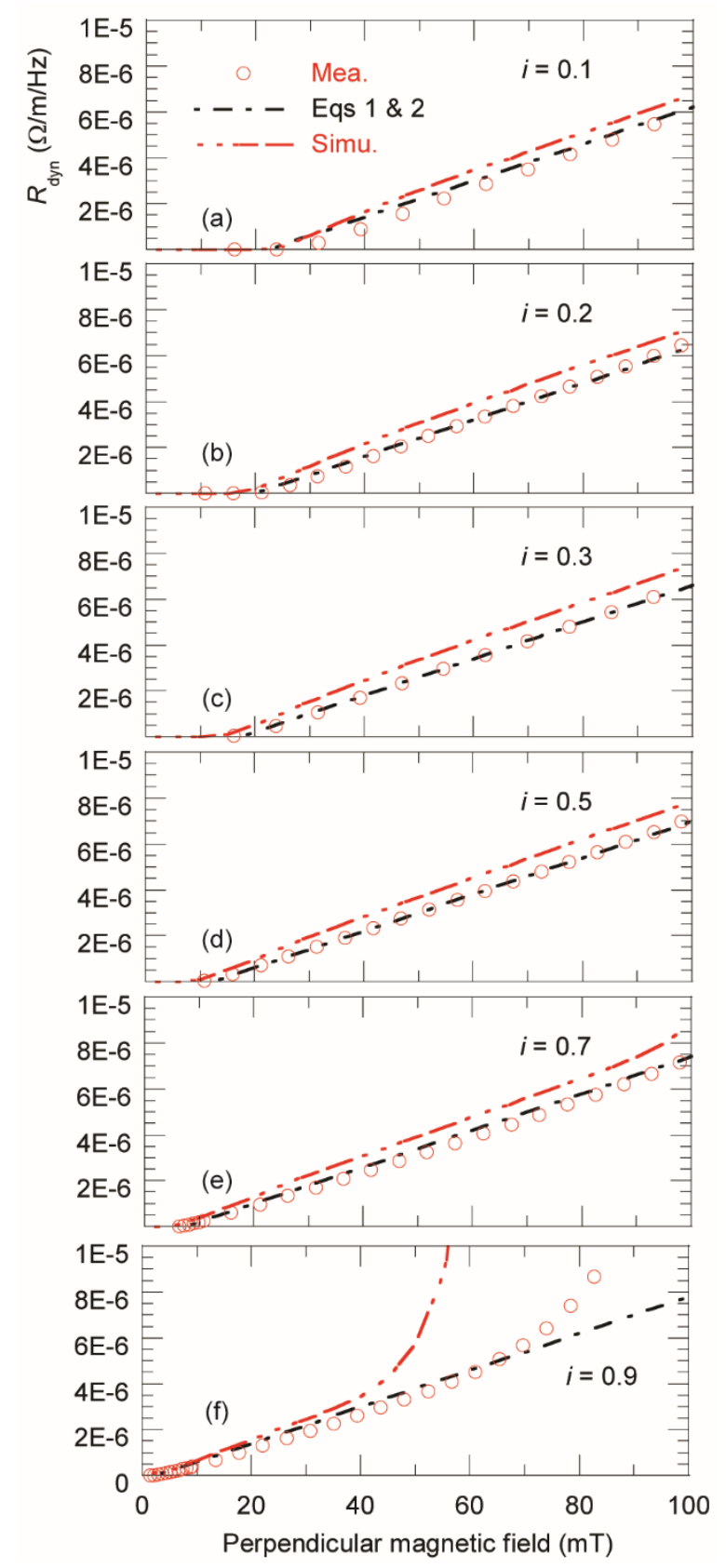

Figure 7. Measured $R_{\mathrm{dyn}}$ values for the sample at $67.89 \mathrm{~Hz}$ for $i=0.1,0.2,0.3,0.5,0.7$, and 0.9 compared with values derived from the combination of equations (1) and (2), and FEM simulation considering effective width of the wire.

Having established the true effective conductor width of the conductor under study, we can now examine the dynamic resistance measured at very low transport currents, where equations (2) and (3) significantly diverge from each other. Figure 8 shows the measured $R_{\mathrm{dyn}}$ values for $i<0.1$, again plotted as a function of $B_{\mathrm{a}, \perp \text {. Once }}$ again, analytical values calculated from equation (1) are plotted alongside the experimental data. In this case the effective conductor width $2 a^{\prime}$ was used, and threshold values were calculated using both equation (2) and (3). It is immediately apparent that at these low transport currents, the calculated values from equation (3) 
show much better agreement with experiment than the equivalent values from equation (2). The deviation between the relative agreement of the two analytical equations becomes increasingly apparent with decreasing $i$, indicating that equation (2) does not appear to be valid at very low values of reduced current, where $i<0.1$. The cause of this divergence is discussed below.

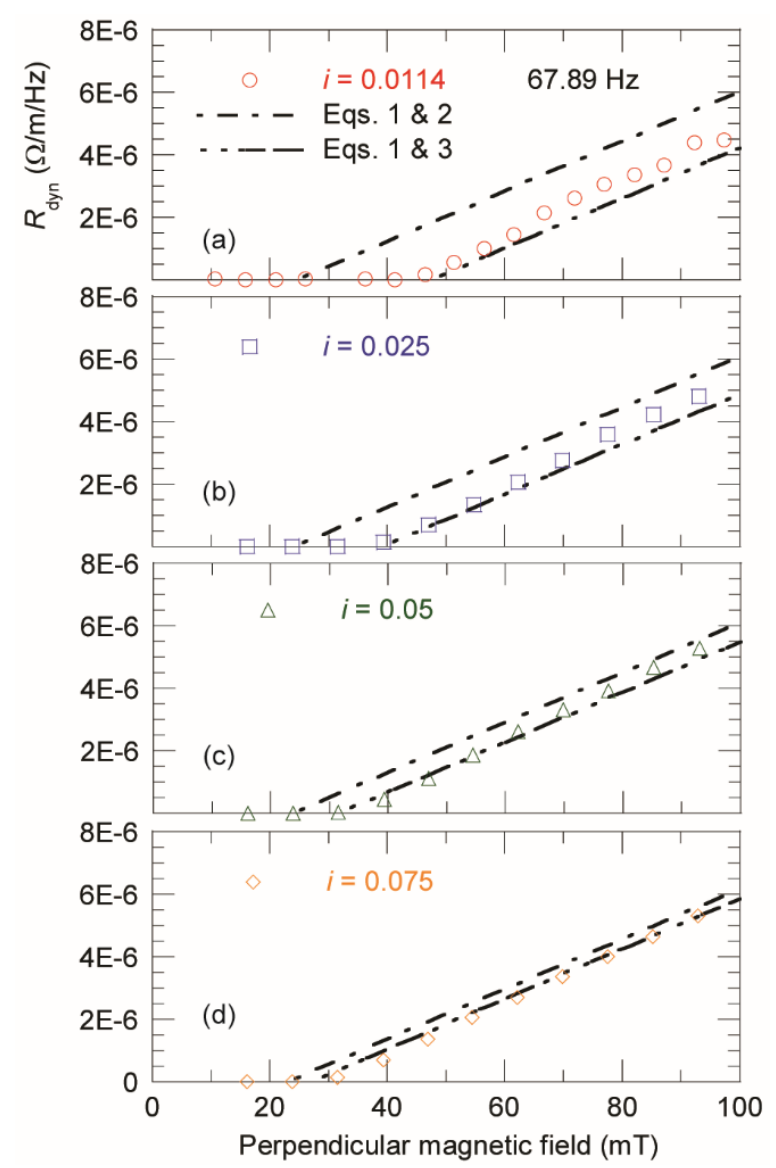

Figure 8. Measured $R_{\mathrm{dyn}}$ values for the sample at $67.89 \mathrm{~Hz}$ for $i=0.0114,0.025,0.05$, and 0.075 compared with values from the combinations of equations (1) and (2) and equations (1) and (3) considering effective wire width 
Figure 9 shows the experimentally-obtained $B_{\mathrm{th}, \perp}$ values plotted as a function of $i$. Also shown are calculated values from both equations (2) and (3), using both the physical and effective conductor widths, $2 a$ and $2 a$,, respectively. For both equations we again see that values calculated using the reduced effective conductor width provide better agreement with experiment, thus providing further confidence in this initial conclusion. It is also clearly apparent that for $i \geq 0.2$, equation (2) yields the best agreement with experiment. This is consistent with our previous work [16], in which we showed that equation (3) consistently underestimates $B_{\text {th }}$ in this technologically-relevant regime. However, in the very low current region where $i \leq 0.1$, it is equally clear that equation (3) provides substantially better agreement with our experimental data. To understand this, we must consider the underlying assumptions behind each equation.

Equation 2 assumes that the 'current reversal zone' is thin and always equal to the value which occurs at the maxima of the Brandt gamma curve [16]. This is valid as long as the frozen flux region (in which the transport current flows) is significantly wider than the current reversal zone. However, at sufficiently low DC transport currents, this assumption does not hold. In this case, the current reversal zone can become compressed by the surrounding shielding currents, resulting in a consequent increase in the effective penetration field. This increase in the gradient of current-reversal leads to a reduction in the dynamic loss, but at the expense of an increase in the magnetisation loss (which is not measured in the experiments reported here). The analysis of [18] accurately describes the non-linear evolution in current and flux profile within the wire and is valid at all values of $i$ - thus equation 3 yields more accurate values of $B_{\mathrm{th}, \perp}$ in the very low current regime. Despite this, equation 3 consistently underestimates $B_{\mathrm{th}, \perp}$ at higher current levels. This is because (3) assumes that the net flux passing the centre of the current reversal zone must always interact dissipatively with the transport current. In fact, at fields just below $B_{\mathrm{th}, \perp}$, flux can exit from the same side of the zone as it entered, leading to zero net dissipation over the full cycle. Equation (2) accounts for this by assuming that all flux is ejected non-dissipatively until the current reversal zone is compressed to the same width as occurs at the maxima of the AC magnetisation loss (the Brandt gamma curve) [16]. Empirically, this delivers better agreement with the experimental data for $i \geq 0.2$, whilst also being advantageously simple to apply.

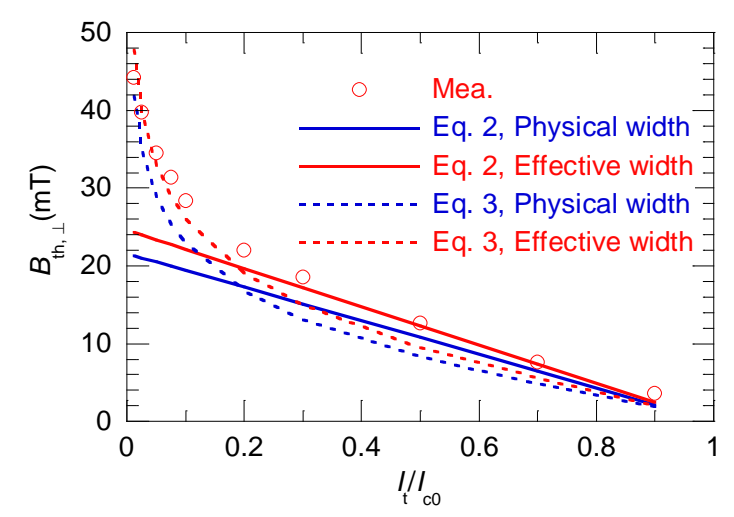

Figure 9. Comparison of $B_{\mathrm{th}, \perp}$ values from experiment and equations (2) and (3) using physical and effective wire width.

\subsection{Angular dependence of $R_{d y n}$ under non-perpendicular alternating magnetic fields}

Figure 10 shows experimentally-measured $R_{\text {dyn }}$ values obtained at ten different angles of applied magnetic field, plotted versus the field amplitude, $B_{\mathrm{a}}$. As before, linear fits of the experimental data are shown, and used to obtain the threshold field values, $B_{\mathrm{th}}$ from the $x$-axis intercepts. For a given value of DC current $i, B_{\mathrm{th}}$ 
decreases with decreasing field angle $\alpha$, whilst the gradient of the fitted linear lines increases with decreasing $\alpha$.

At $i=0.9$ and for low field angle we once again observe a non-linear contribution to $R_{\mathrm{dyn}}$ at high field amplitudes. Similarly to the perpendicular field data, we attribute this to flux flow resistance arising once $I_{\mathrm{c}}\left(B_{\mathrm{a}}\right)<I_{\mathrm{t}}[16]$. It is apparent that this non-linear contribution decreases rapidly with increasing field angle such that 'lift-off' from the linear trend is negligible once $\alpha \geq 50^{\circ}$. This reflects the lower sensitivity of $I_{\mathrm{c}}$ to non-perpendicular magnetic fields.

We do not observe any measurable values of $R_{\mathrm{dyn}}$ under parallel magnetic field ( $\alpha=90^{\circ}$ ) at any of the transport currents studied, except for an extremely small onset at $i=0.9$ and $B_{\mathrm{a}}>80 \mathrm{mT}$. This implies that parallel magnetic field plays almost no role in the dynamic resistance of the coated conductor, which is entirely different to the case for BSCCO wires $[4,40]$. The perpendicular component of the applied magnetic field can be expressed as $B_{\mathrm{a}, \perp}=B_{\mathrm{a}} \cos \alpha$, meaning that the threshold value at angle $\alpha$, can similarly be expressed as $B_{\mathrm{th}}(\alpha)=B_{\mathrm{th}, \perp} /(\cos \alpha)$. As such, we expect $B_{\mathrm{th}}(\alpha)$ to increase with increasing $\alpha$, and this is consistent with the trend observed in figure 10. Similarly, the gradient $\mathrm{d} R_{\mathrm{dyn}} / \mathrm{d} B_{\mathrm{a} \perp}$ in equation (1) now becomes $\mathrm{d} R_{\mathrm{dyn}} / \mathrm{d} B_{\mathrm{a}}=\left(4 a^{\prime} \cdot \cos \alpha\right) / I_{\mathrm{c} 0}$, which is also consistent with the observed trend in this figure. 
1

2

3

4

5

7

8

9

10

11

12

13

14

15

16

17

18

19

20

21

22

23

24

25

26

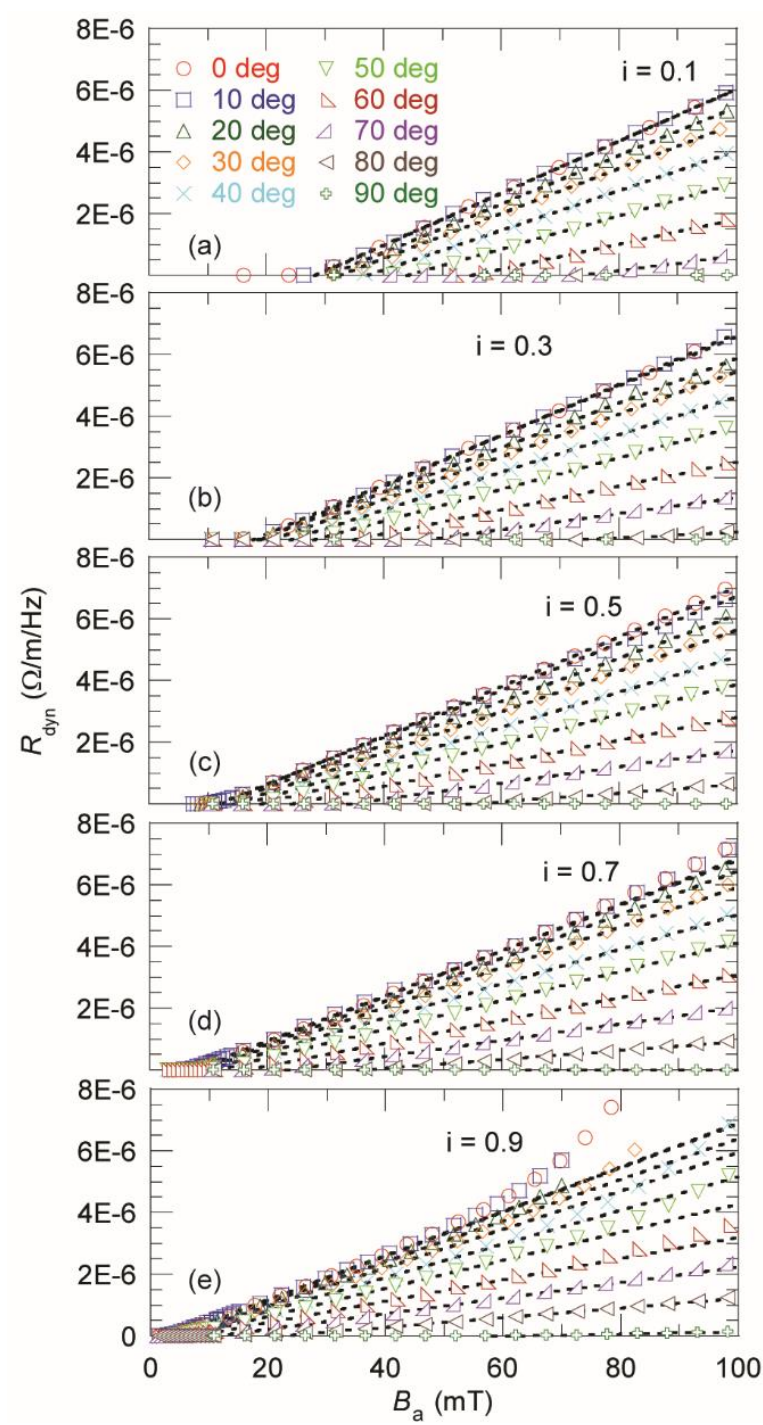

Figure 10. Measured $R_{\mathrm{dyn}}$ values at various field angles plotted as a function of applied magnetic field amplitude at $67.89 \mathrm{~Hz}$ (a) $i=0.1$, (b) $i=0.3$, (c) $i=0.5$, (d) $i=0.7$, and (e) $i=0.9$.

Figure 11 shows fitted values of $B_{\mathrm{th}}(\alpha)$ obtained from the experimental data at various angles, and plotted as a function of the reduced current, $i$. The dotted lines show calculated values using equation (2) for $i \geq 0.2$ (taking $B_{\mathrm{th}}=B_{\mathrm{th}, \perp} /(\cos \alpha)$ ), and using equation (3) for $i<0.2$ (taking $B_{\mathrm{th}}=B_{\mathrm{th}, \mathrm{MB}} /(\cos \alpha)$ ). Excellent agreement is obtained between the experimental and calculated values of $B_{\text {th }}$ for all $\alpha<80^{\circ}$, indicating that the threshold field is determined solely by the perpendicular field component of the applied field. The small perpendicular field component at $\alpha=80^{\circ}$ results in a large threshold field ( $80 \mathrm{mT}$ at $i=0.3$ ). This meant that there were few data points available to perform linear fits to the experimental data (see figures 10 (a), (b) and (c)). As a result, the fitted values obtained at this field angle must be treated with a little caution, and this is the likely cause of the divergence between calculated and experimental values observed at this nearlyparallel field angle. 


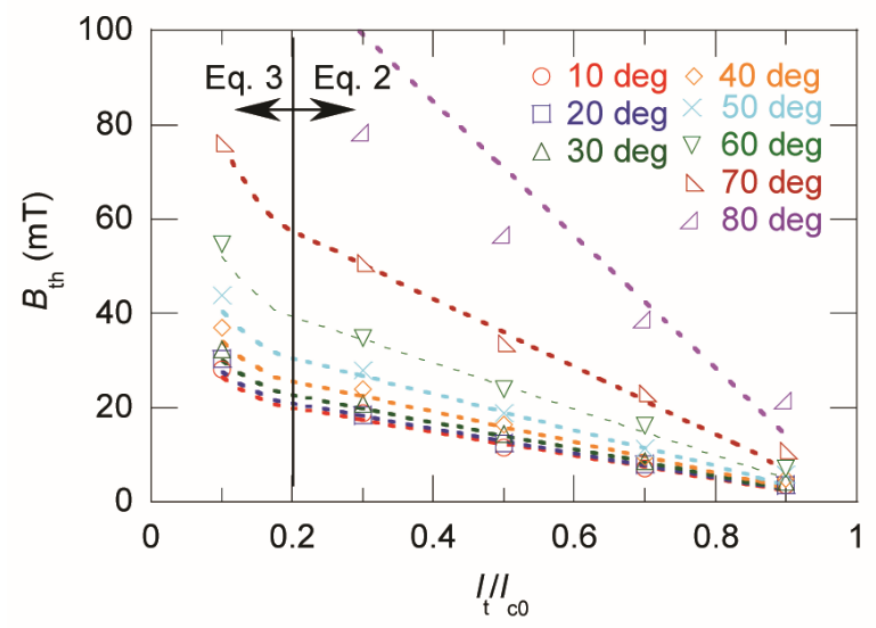

Figure 11. Experimentally-determined values for the threshold magnetic field, $B_{\text {th }}$ obtained for various field angles, $\alpha$, and plotted versus the reduced transport current, $i=I_{t} / I_{\mathrm{c} 0}$. Dotted lines show calculated values using $B_{\mathrm{th}}=B_{\mathrm{th}, \perp} /(\cos \alpha)$ for $i \geq 0.2$ (from equation 2), and $B_{\mathrm{th}}=B_{\mathrm{th}, \mathrm{MB}} /(\cos \alpha)$ for $i<0.2$ (from equation 3).

Finally, in figure 12, the measured $R_{\text {dyn }}$ values (shown previously in figure 10) are now re-plotted as a function of the perpendicular component of the applied magnetic field, $B_{\mathrm{a}, \perp}=B_{\mathrm{a}} \cos \alpha$. The entire experimental dataset is shown, together with the values calculated from equations (1) and (2). The $R_{\text {dyn }}$ values measured at each different field angle now collapse onto a common curve, and show excellent agreement with the analytically calculated values for all linear regions shown. Taken together, the results in figures 11 , and 12 provide compelling evidence that (for all $\alpha \leq 80^{\circ}$ ), the dynamic resistance the dynamic resistance of this coated conductor wire is determined solely by the perpendicular component of the applied magnetic field. 
1

2

3

4

5

6

7

8

9

10

11

12

13

14

15

16

17

18

19

20

21

22

23

24

25

26

27

28

29

30

31

32

33

34

35

36

37

38

39

40

41

42

43

44

45

46

47

48

49

50

51

52

53

54

55

56

57

58

59

60

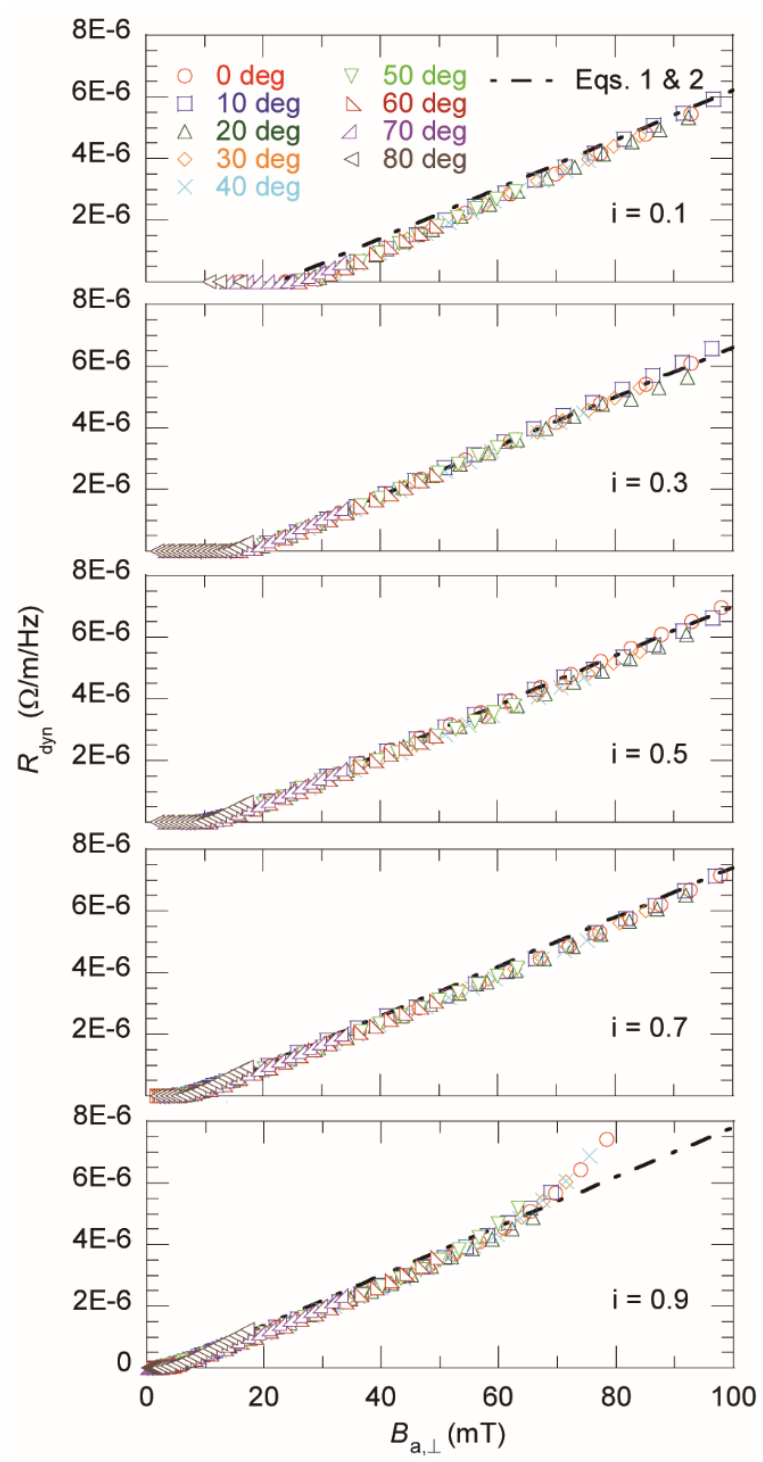

Figure 12. Measured $R_{\mathrm{dyn}}$ values at various field angles plotted as a function of perpendicular magnetic field component, $B_{\mathrm{a}, \perp}=B_{\mathrm{a}} \cos \alpha$. Data obtained at $67.89 \mathrm{~Hz}$ for (a) $i=0.1$, (b) $i=0.3$, (c) $i=0.5$, (d) $i=0.7$, and (e) $i$ $=0.9$. 


\section{Conclusion}

In this work, we have measured dynamic resistance in a $4 \mathrm{~mm}$-wide YBCO coated conductor carrying DC currents under AC magnetic fields incident at various different field angles. Measurements were carried out using a wide range of DC currents from $0.0114 \leq i \leq 0.9$, and the applied field angle was changed from zero to $90^{\circ}$ with a resolution of $10^{\circ}$.

When the conductor width was taken to be the measured physical dimension of the wire, we found that experimentally-measured $R_{\text {dyn }}$ values did not agree well with calculated analytical values, nor with the numerical 2D-FEM results computed using the $T$ - method, nor with transport AC loss measurements of the same wire. We have attributed this difference to a non-uniform critical current distribution at the edges of the coated conductor wire, which results in a reduced effective conductor width. To account for this, we have calculated an equivalent conductor width from the measured gradient, $d R_{\mathrm{dyn}} / d B_{\mathrm{m}}$ for all measured values at $i$ $>0.1$. This value was confirmed through independent measurements of the AC transport loss of this wire. Using this effective conductor width, we then obtain substantially better agreement between experiment and both our analytical and 2D-FEM calculations.

We have compared our experimental data with two different analytical expressions which have been proposed to describe the threshold field under perpendicular fields. Interestingly, we find that the relative agreement between these two equations and experiment changes depending on the magnitude of the reduced current, $i$. For $i \geq 0.2$, we find that the simple linear expression within equation (2) provides best agreement with experiment, which is consistent with previously reported results in [16]. This high current regime is technologically important, as it corresponds to situations in which most of current-carrying capacity of the wire is being employed. However, at very low DC current levels $(i \leq 0.1)$ we find that experimental values of $B_{\text {th }}$ increase sharply with decreasing $i$ and are thus best described by the equation of Mikitik and Brandt (equation (3)). We believe that our results represent the first robust experimental confirmation of the work presented in [18] for the low DC current regime.

We have also measured the dynamic resistance which occurs at non-perpendicular applied field angles. We have found that this is dominated solely by the perpendicular field component. This means that $R_{\mathrm{dyn}}$ can be simply calculated using solely the expressions which describe the perpendicular field behaviour (unless the field angle is close or equal to $90^{\circ}$ ). The values of $R_{\text {dyn }}$ measured under parallel magnetic fields are more than three orders of magnitude smaller than those observed under perpendicular field, and this renders these values negligible in most practical applications. From an experimental standpoint, such extremely small DC signals are challenging to measure, and the measured value is highly sensitive to minor misalignment of the sample in the applied field. As such, precise determination of the parallel field behaviour of coated conductor wires remains a largely unexplored avenue for further future work.

\section{Acknowledgments}

Z. Jiang acknowledges Gennady Sidorov for his assistance in the development of the specialist cryostat used in this work, and Wenjuan Song for transport AC loss measurement of a SuperPower wire sample. 


\section{References}

[1] Majkic G, Galstyan E, and Selvamanickam V 2015 IEEE Trans. Appl. Supercond. 256605304.

[2] Andrianov V V, Zenkevitch V B, Kurguzov V V, Sytchev V V, and Ternovskii F F 1970 Sov. Phys. JETP. 31815.

[3] Ogasawara K, Yasukochi K, Nose S, and Sekizawa H 1976 Cryogenics 1733.

[4] Oomen M P, Rieger J, Leghissa M, ten Haken B, and ten Kate H H J 1999 Supercond. Sci. Technol. 12 (1999) 382.

[5] Pardo E 2013 IEEE Trans. Appl. Supercond. 244700105.

[6] Kalsi S 2011 Application of high temperature superconductors to electric power equipment (IEEE Press) pp 96.

[7] Sung H J, Go B S, Jiang Z, Park M, and Yu I K 2016 Physica C 530 133-7.

[8] Bumby C W, Badcock R A, Sung H J, Kim K M, Jiang Z, Pantoja A E, Bernado P, Park M, and Buckley R G 2016 Supercond. Sci. Technol. 29024008.

[9] Jiang Z, Hamilton K, Amemiya N, Badcock R A, and Bumby C W 2014 Appl. Phys. Lett. 105 112601 .

[10] Geng J and Coombs T A 2015 Appl. Phys. Lett. 107142601.

[11] Jiang Z, Bumby C W, Badcock R A, Sung H J, Long N J, and Amemiya N 2015 Supercond. Sci. Technol. 28115008.

[12] Bumby C W, Jiang Z, Storey J G, Pantoja A E, and Badcock R A 2016 Appl. Phys. Lett. 108 122601.

[13] Pardo E 2017 Supercond. Sci. Technol. 30060501.

[14] Ciszek M, Tsukamoto O, Ogawa J, and Miyagi D, 2002 AIP Conf. Proc. 614606.

[15] Duckworth R C, Zhang Y F, Ha T, and Gouge M J 2011 IEEE Trans. Appl. Supercond. 213251.

[16] Jiang Z, Toyomoto R, Amemiya N, Zhang X, and Bumby C 2017 Supercond. Sci. Technol. 30 03LT01.

[17] Jiang Z, Toyomoto R, Amemiya N, Bumby C, Badcock R, and Long N 2017 IEEE Trans. Appl. Supercond. 275900205.

[18] Mikitik G P and Brandt E H, 2001 Phys. Rev B 64092502.

[19] Brandt E H and Indenbom M 1993 Phys. Rev. B 43 12893-906.

[20] Zhou W, Staines M, Jiang Z, Badcock R A, Long N J, and Fang J "Shielding of eddy current loss in metal layers of REBCO superconducting tapes and Roebel cables" Submitted to IEEE Trans. Appl. Supercond.

[21] Li Q, Yao M, Jiang Z, Bumby C W, and Amemiya N "Numerical modelling of dynamic loss in HTS coated conductors under perpendicular magnetic fields" Submitted to IEEE Trans. Appl. Supercond.

[22] Jiang Z and Amemiya N 2004 Supercond. Sci. Technol. 17 371-9.

[23] Fukui S, Kitoh Y, Numata T, Tsukamoto O, Fujikami J, and Hayashi K 1998 Adv. Cryogenic. Eng. 44723.

[24] Carpenter C J, 1977 Proc. IEE, 124, 1026-34.

[25] Preston T W and Reece A B J, 1982 IEEE Trans. Magn. 18, 486-91.

[26] Nakata T, Takahashi N, Fujiwara K, and Okada Y, 1988 IEEE Trans. Magn. 24 94-7.

[27] Jiang Z, Long N, Staines M, Li Q, Slade R, Amemiya N, and Caplin A, 2012 IEEE Trans. Appl. Supercond., 228200306.

[28] Li Q, Amemiya N, Takeuchi K, Nakamura T, and Fujiwara N, 2011 IEEE Trans. Appl. Supercond. 21 953-6.

[29] Li Q, Amemiya N, Nishino R, Nakamura T, and Okuma T, 2013 Physica C, 484 217-22.

[30] Li Q, Xin Y, and Wang S, 2017 IEEE Trans. Appl. Supercond. 265900205.

[31] Tsukamoto O 2005 Supercond. Sci. Technol. 18 596-605.

[32] Jiang Z, Amemiya N, Maruyama O, Shiohara Y 2007 Physica C 463-465 790-4. 
[33] Amemiya N, Jiang Z, Kato T, Ohmatsu K, Masuda T, and Shiohara Y 2009 Physica C 469 1427-31.

[34] Li Q, Amemiya N, Kakeuchi K, Nakamura T, and Fujiwara N 2010 Supercond. Sci. Technol. 23 (2010) 115003.

[35] Norris W T 1970 J. Phys. D: Appl. Phys. 3 489-507.

[36] Doan N N, Grilli F, Ashworth S P, and Willis J O 2009 Supercond. Sci. Technol. 22055014.

[37] Brambilla R, Grilli F, Doan N N, Martini L, and Sirois F 2009 Supercond. Sci. Technol. 22075018.

[38] Higashikawa K, Katahira K, Okumura K, Shiohara K, Inoue M, Kiss T, Shingai Y, Konishi M, Ohmatsu K, Yoshizumi M, and Izumi T 2013 IEEE Trans. Appl. Supercond. 236602704.

[39] http://www.superpower-inc.com/system/files/SP_2G+Wire+Spec+Sheet_2014_web_v1_0.pdf (as of 10 July 2017).

[40] Ciszek M, Knoopers H G, Rabbers J J, ten Haken B, and ten Kate H H J 2002 Supercond. Sci. Technol. 151275. 